\title{
AN AUDIT OF NEONATAL AUTOPSIES: PREDICTORS OF FAILURE
}

\author{
V. Sundaram, P. Kumar, S. Saini
}

\section{Pediatrics, Postgraduate Institute of Medical Education and Research, Chandigarh, India}

Autopsy rates in neonates are dismally low across the world. We did an audit of the current status of neonatal autopsies and the factors associated with failure of neonatal autopsies in a Level III neonatal unit over 10 months. The treating team was requested to complete a pre-tested questionnaire after death and autopsy counseling. Putative risk factors were then compared between autopsied versus non-autopsied. Out of 100 neonatal deaths a complete autopsy was performed in $50(50 \%)$. The mean gestation and birth weight were $32.1 \pm 4.2$ weeks and $1397 \pm 612 \mathrm{~g}$ respectively. The median age of death was 33 hours. The death was declared in an open area in front of the NICU in $58(83 \%)$ deaths. A larger family size, grand father headed family, an extreme emotional response to death declaration as well as to autopsy counseling and a longer death to autopsy counseling time were significantly more common in the non-autopsied group. Refusal by an elder member of the family was the most common reason for denial. A larger family size [OR 10.1 (95\% CI 1.7, $67), p=01$ ] and a delay in autopsy counseling following death [OR 9.8 (95\% CI 2.3, 42), $\mathrm{p}=0.002$ ] were independent predictors of failure to give autopsy consent. A low neonatal autopsy rate of $50 \%$ was observed in this audit. Modification of certain epidemiological factors like decreasing the time between death and autopsy counseling may increase the autopsy rates. A larger nationwide audit is warranted to improve the neonatal autopsy rates. 